Hinführung 


\title{
Christliche Tierethik - Themen und Diskurse
}

\author{
Martin M. Lintner
}

\section{Das Christentum und die Tiere}

Christentum und Tiere? Franz von Assisi, dessen Predigt auch Vögel gelauscht haben sollen und der jedes Geschöpf als Bruder oder Schwester angesprochen hat, und Albert Schweizer, der sich mit jedem Lebewesen durch Wesensverwandtschaft und Schicksalsgemeinschaft verbunden wusste, sind Namen, die damit assoziiert werden. Vielleicht noch Philipp Neri, der Katzen und Hunde liebte und aus Tierliebe kein Fleisch aß, oder - zumal in der katholischen Tradition - der eine bzw. die andere Heilige, dessen bzw. deren Attribut ein Tier ist: die Evangelisten Markus, Lukas und Johannes mit einem Löwen, Stier bzw. Adler; Antonius, der Mönchsvater, mit einem Schwein und weiteren Wildtieren; Hieronymus mit dem Löwen, aus dessen Pfote er, so die Legende, einen Dorn entfernt hatte; Leonhard, der Patron des Bauernstandes, mit einem Rind; Agnes mit dem Lamm; Hubertus, der Patron der Jäger, mit dem Hirsch; Kolumban und Korbinian, ebenso Romedius von Thaur mit einem gezähmten Bär etc. Die Tiere als Attribute von Heiligen symbolisieren jedoch meistens eine Charaktereigenschaft oder ein Ereignis aus dem Leben dieser Menschen, sie spiegeln in der Regel nicht ihre Beziehung zu den dargestellten Tieren wider. In der Volksfrömmigkeit hat sich die Darstellung von Heiligen mit Tieren nicht unbedingt in einem verantwortungsbewussten Umgang der Menschen mit den jeweiligen Tieren niedergeschlagen. Eher haben „katholische Bildsymbolik, kirchliches Brauchtum und religiöse Feste die traditionelle Lehre von der Herrschaft des Menschen über die Tiere tief im kollektiven Gedächtnis vieler Katholiken verankert" (Kurt Remele 2016, 109-110). Tatsächlich ist das Christentum weitgehend geprägt von philosophischen Einflüssen und einer Tradition der Bibelauslegung, die den Menschen gegenüber den Tieren eine Vorrangstellung einräumt, die als Legitimation dafür gedient hat, Tiere auf der dinglichen Ebene anzusiedeln und als Ressource zu nutzen. Im Lauf der christlichen Tradition hat eine Weltdeutung und Haltung gegenüber den Tieren Oberhand gewonnen, die Papst Franziskus in seiner Umweltenzyklika Laudato si' (2015) als „despotischen Anthropozentrismus“ anprangert, „der sich nicht um die anderen 
Geschöpfe kümmert“ (Nr. 68). Seitens der Theologie entspricht dem eine weitgehende "Tiervergessenheit", die selbst in den gegenwärtigen Abhandlungen über Schöpfungstheologie zu konstatieren ist (Julia Enxing 2021) und die es zu überwinden gilt (Rainer Hagencord ${ }^{4} 2009$; Simone Horstmann/Thomas Ruster/Gregor Taxacher 2018; Simone Horstmann 2020). Die Theologie muss sich auch der kritischen Frage stellen, inwiefern nicht nur philosophische Traditionen wie die Vorstellung der Tiere als vernunftlose Lebewesen oder der cartesianische Dualismus, der ein mechanistisches Tierbild prägte, sondern auch das Christentum mit der Anschauung der Überlegenheit des Menschen über die Tiere sowie dem religiös begründeten, vielfach falsch ausgelegten Ermächtigungsauftrag, über die Tiere zu herrschen, mitverantwortlich ist für die massenhafte, auch strukturelle Gewalt gegenüber Tieren (Simone Horstmann [Hg.] 2021).

Der Tierschutz stellt bis heute kein Kernanliegen der christlichen Kirchen dar. In Vergessenheit geraten sind Persönlichkeiten wie Philipp Jakob Spener (1635-1705), Laurids Smith (1754-1794), Christian Adam Dann (1758-1837), Albert Knapp (1798-1864) und viele andere (Hans-Eberhard Dietrich 2019, 95-109; Stefan Schleißing/Herwig Grimm 2012, 48-49). Der lutherisch-pietistische Theologe Philipp Jakob Spener gab in einer Katechese über das biblische Tötungsverbot zu bedenken, dass es nicht nur auf den Menschen zu beziehen sei, sondern vielmehr verbiete, irgendeine Kreatur unnütz und mutwillig zu quälen oder zu töten. Ein achtloser und grausamer Umgang mit den Tieren widerspreche dem Willen des Schöpfers. Der Mensch, so Spener in einer Katechese über das „Seufzen der Schöpfung“ in Röm 8,19-24, solle sich hüten, ein Tier so zu behandeln, dass es seufzen müsse und Anlass habe, sich vor Gott über den Menschen zu beklagen. Der protestantische dänische Philosoph und Theologe Laurids Smith vertrat im Buch Versuch eines vollständigen Lehrgebäudes der Natur und Bestimmung der Thiere und der Pflichten des Menschen gegen die Thiere (Erst- und Zweitauflage 1793) die These, dass den Tieren eine eigene geschöpfliche Würde zukomme. Sie hätten Rechte, vergleichbar mit jenen des Menschen, und es sei menschliche Pflicht, sie zu achten. Der pietistische Pfarrer Christian Adam Dann veröffentlichte zwei wichtige Aufsätze, deren Titel eine deutliche Ansage sind: Bitte der armen Thiere, der unvernünftigen Geschöpfe, an ibre vernünftigen Mitgeschöpfe und Herrn, die Menschen (Erstauflage 1822, Zweitauflage 1838) und Nothgedrungener, durch viele Beispiele beleuchteter Aufruf an alle Menschen von Nachdenken und Gefühl $z u$ gemeinschaftlicher Beherzigung und Linderung der unsäglichen Leiden der in unserer Umgebung lebenden Thiere (1832) (Martin H. Jung [Hg.] 2002). Anlass der ersten Schrift war ein Ereignis im Sommer 1821, das den Autor zutiefst verstört und empört hatte. Er fand eines Tages einen Storch, der seit 
Jahren auf seinem Kirchdach seinen Nistplatz hatte, durchsiebt von Schrotkugeln. Das Storchenpaar hatte er wiederholt in seinen Predigten als Vorbild für lebenslange Treue erwähnt. Erbost über die sinnlose Tötung des Storches verfasste er eine flammende Schrift gegen Tierquälerei und für einen sorgsamen Umgang mit den Tieren. Darin prangerte er nicht nur die sinnlose Quälerei von Haus-, Nutz- und Wildtieren sowie die Vernachlässigung und Misshandlung des Viehs, sondern auch Tierversuche und das Ausplündern der Natur durch Eierdiebstahl und Vogelfang an. Dann bekräftigte seine Position mit vielen Zitaten aus der Bibel, die von Gottes barmherzigem Umgang mit den Tieren berichten und vom gerechten Menschen verlangen, sich um das Wohl der Tiere zu kümmern. Er rief dazu auf, die Tiere als von Gott geliebte Mitgeschöpfe zu achten. Ein Mensch, der Tiere quäle, könne auch Gott nicht lieben. Der Begriff „Mitgeschöpf“ dürfte inspiriert sein von Martin Luthers Auslegung des Glaubensbekenntnisses im Kleinen Katechismus: „Ich glaube, dass mich Gott geschaffen hat samt allen Kreaturen" (Art. 1). Luther, der selbst einen Hund namens „Tölpel“ besaß, hat auch laut darüber nachgedacht, ob Tiere in den Himmel kommen. In einer Tischrede soll er zu jemandem, der um seinen Hund getrauert hat, gesagt haben, dieser werde in der neuen Schöpfung, wo Gott alles in allem sei, eine goldene Haut und Haare aus Perlen haben (Ulrich Seidel 2017, 58). Diese doch etwas überspitzte Redeweise lässt jedoch Zweifel aufkommen, wie ernst oder polemisch es Luther gemeint hat. Zurück zu Christian Adam Dann: Dessen Freund Albert Knapp, ebenso ein pietistischer Pfarrer, der bereits an Danns zweiter Schrift mitgewirkt hatte, gründete 1837 in Baden-Württemberg den ersten Tierschutzverein Deutschlands. Gleichgesinnte gründeten weitere Vereine in ganz Deutschland und bereits 1839 wurde das erste württembergische Tierschutzgesetz erlassen sowie das Verbot der Tierquälerei ins Strafgesetzbuch aufgenommen. 1871 wurden erstmals Straftaten gegen Tiere im Reichsstrafgesetzbuch definiert und 1891 als Dachverband der bis dahin bestehenden Tierschutzvereine der Deutsche Tierschutzbund gegründet.

Trotz dieser Pioniere aus der lutherischen Tradition, die eine wichtige Vorreiterrolle für die Entwicklung des Tierschutzes und die Gründung von Tierschutzvereinen gespielt haben, bleibt das Verhältnis der christlichen Kirchen zum Tierschutz bis heute ambivalent. Weder die evangelische noch die katholische Kirche haben sich tierethische Fragen als ein vordergründiges Anliegen zu eigen gemacht. Es gibt zwar Initiativen wie die Aktion Kirche und Tiere e. V. (AKUT), allerdings "nur“ als Arbeitskreis 
von Menschen aus den Kirchen, die sich aus persönlicher Überzeugung für die Tiere einsetzen. ${ }^{1}$

\section{Kirchliche Positionen}

Die Evangelische Kirche in Deutschland (EKD) bringt sich schon seit Längerem engagiert in die Debatten um ethische Aspekte in der Nutztierhaltung aus christlicher Perspektive ein (Stefan Schleißing/Herwig Grimm 2012, 45-66). Bereits 1991 legte der Wissenschaftliche Beirat des Beauftragten für Umweltfragen des Rates der EKD den Diskussionsbeitrag Zur Verantwortung des Menschen für das Tier als Mitgeschöpf vor. Darin heißt es:

„Mensch und Tier gehören zusammen als Geschöpfe: Beide geben sie sich ihre Lebensmöglichkeiten, ihren Lebensraum und ihre Lebensversorgung, nicht selbst. Sie verdanken ihr Leben Gott, dem Schöpfer und Erhalter. Das schließt sie zusammen in Abhängigkeit und Angewiesensein (Ps 104,27-30) und verwehrt dem Menschen, sich in Hochmut grundsätzlich vom Tier abzusetzen und über es zu erheben. [...] Vor allem aber haben die Mitgeschöpfe der Menschen unabhängig von ihrem Nutzwert einen eigenen Sinn und Wert“ (ebd., 5 und 7).

Das Papier wurde bewusst als ein Beitrag zur kontrovers geführten Auseinandersetzung um die Ausrichtung des evangelisch-kirchlichen Einsatzes im Tierschutz verstanden. Es sollte helfen, mehr Klarheit darüber zu finden,

„ob das Ziel der Verminderung der Gewalt zwischen Mensch und Tier vom biblischen Zeugnis her weitergeführt werden darf zu der radikalen Position einer prinzipiellen Ablehnung von Gewalt, anders gesagt: in welcher Weise ein Schöpfungspazifismus eschatologische Hoffnung bleiben muss und wieweit er schon jetzt das Handeln leiten soll“" (ebd., Vorwort).

2019 veröffentlichte die Kammer für nachhaltige Entwicklung der EKD das Positions- und Impulspapier Nutztier und Mitgeschöpf? Tierwobl, Ernährungsethik und Nachhaltigkeit aus evangelischer Sicht. ${ }^{2}$ Das Dokument setzt sich zum Ziel,

1 Siehe dazu die Webseite des Vereins: aktion-kirche-und-tiere.de (abgerufen am 02.07.2020).

2 Im Dokument wird bemängelt: „Obwohl das Thema Tierwohl und Tierethik eine so hohe Relevanz in der Gesellschaft hat, haben sich die Kirchen sehr lange nicht mehr dazu geäußert. Die letzte Verlautbarung der EKD dazu mit dem Titel ,Zur 
„sich nicht nur mit wichtigen Einzelfragen und Regelungsbereichen zu befassen, sondern auch die grundsätzlichen Fragen des Verhältnisses zwischen Mensch und Tier zu beleuchten, wie sie insbesondere im Diskurs über neue Ansätze der Nutztierethik reflektiert werden“ (ebd., 8).

Aus bibeltheologischer Perspektive kommt die EKD zum Schluss, dass

„man biblisch sachgemäß durchaus von einer eigenen, Würde der Tie$\mathrm{re}^{6}$ als Mitgeschöpfe des Menschen sprechen kann, auch wenn es biblisch gesehen keine wirklich entfaltete Lehre von der ,Tierwürde‘ gibt und das Prädikat der ,Ebenbildlichkeit' dem Menschen vorbehalten bleibt“" (ebd., 13).

Die EKD fordert daher eine höhere Wertschätzung für Tiere sowie die Verbesserung der Situation der Nutztierhaltung, die als „umfassender zivilisatorischer Umlernprozess, der dem Prozess der Dekarbonisierung unserer Weltwirtschaft in nichts nachsteht“ (ebd., 9), bezeichnet wird.

Die (katholische) Deutsche Bischofskonferenz hat 1993 die Arbeitshilfe Die Verantwortung des Menschen für das Tier herausgegeben. ${ }^{3}$ Darin wird anerkannt, dass der Tierschutz „immer mehr als eine sittliche Verpflichtung empfunden wird“:

„Immer schon waren Haustiere Gefährten des Menschen. Sie sind lebende und fühlende Mitgeschöpfe des Menschen, die einen Eigenwert in Gottes guter Schöpfung haben. Deshalb wird Tierschutz heute immer mehr als eine sittliche Verpflichtung empfunden. Weil wir den Tieren gegenüber eine Verantwortung und Sorgfaltspflicht haben, müssen wir mit ihnen artgerecht umgehen. So ergeben sich z. B. Fragen nach der Berechtigung und den Grenzen von Tierversuchen, Gentechnologie in der Tierzucht, aber auch nach Massentierhaltung und Patentierung von Tieren“ (ebd., 5).

Die katholischen Bischöfe wählen einen verantwortungsethischen Zugang. Einerseits sprechen sie von der „Mitgeschöpflichkeit“ der Tiere und verste-

Verantwortung des Menschen für das Tier als Mitgeschöpf"stammt aus dem Jahr 1991. Auch in der theologischen Ethik und in der Diskussion der weltweiten Ökumene wurden in den letzten zwanzig Jahren die Fragen der Tierethik kaum behandelt" (ebd., 7).

3 Das Heft beinhaltet neben Anregungen für die Praxis (Religionsunterricht, Gemeindearbeit, Liturgie) auch eine Dokumentation von einschlägigen Auszügen aus kirchlichen Erklärungen. 
hen darunter „unsere Gemeinsamkeit mit den Tieren im Strom der lebenden Schöpfung“ (ebd., 9), andererseits betonen sie die Vernunftbegabung des Menschen und gehen ausdrücklich von einem „weitgehenden Konsens" darüber aus, dass er das einzige vernunftbegabte Wesen ist (ebd., 8). Verantwortlich handeln, so die Bischöfe, bedeutet die Verwirklichung von Werten und Gütern, wobei bei konkurrierenden Werten dem je höheren der Vorrang zu geben sei. Die dadurch zu begründende Rangordnung lasse eine Höhergewichtung von menschlichen gegenüber tierlichen Interessen zu, wie aus den angeführten Konkretionen (Tierversuche, Nutztierhaltung, Gentechnik) deutlich wird (ebd., 10-15). Des Weiteren führen die Bischöfe die Handlungsmotivation, die Wahl angemessener Mittel und die Berücksichtigung der beabsichtigten wie unbeabsichtigten absehbaren Folgen ins Feld. Sie kommen zum Schluss, dass

„sich überall dort, wo Tiere ohne gerechten Grund leiden, ethische Bedenken zu erheben haben. Die Bestimmung dessen aber, was ein gerechter Grund ist, kann letztlich nur im Rahmen eines Modells der Verantwortungsethik für bestimmte Fallgruppen oder gar Fall für Fall entschieden werden" (ebd., 15).

Im Wesentlichen findet sich diese Position auch im Katechismus der Katholischen Kirche wieder. Dieser geht an zwei Stellen auf die Tiere bzw. auf die Beziehung der Menschen zu den Tieren ein: einmal im Kontext der Ausführungen über das Glaubensbekenntnis, konkret über die sichtbare Welt der Schöpfung (Nrn. 339-344, 353-354), sodann im Rahmen der Erläuterungen zum Dekalog, konkret zum siebten Gebot „Du sollst nicht stehlen“ (Nrn. 2415-2418, 2456-2457). Zwar heißt es, dass

„jedes Geschöpf seine eigene Güte und Vollkommenheit besitzt [...]. Die unterschiedlichen Geschöpfe widerspiegeln in ihrem gottgewollten Eigensein, jedes auf seine Art, einen Strahl der unendlichen Weisheit und Güte Gottes. Deswegen muss der Mensch die gute Natur eines jeden Geschöpfes achten und sich hüten, die Dinge gegen ihre Ordnung zu gebrauchen. Andernfalls wird der Schöpfer missachtet und es entstehen für die Menschen und ihre Umwelt verheerende Folgen“ (Nr. 339).

Der Grundtenor ist jedoch, dass Gott „alle materiellen Geschöpfe zum Wohl des Menschengeschlechtes bestimmt hat" (Nr. 353). Die Behandlung der Tiere im Rahmen des siebten Gebotes, konkret im Kapitel „Achtung der Menschen und ihrer Güter“, spiegelt diese Position wider. Zwar schuldet der Mensch den Tieren „Wohlwollen“, da sie als „Geschöpfe Gottes seiner fürsorgenden Vorsehung unterstehen [und] schon allein durch ihr Dasein 
Gott preisen und verherrlichen“ (Nr. 2416), doch „Tiere, Pflanzen und leblose Wesen sind von Natur aus zum gemeinsamen Wohl der Menschheit von gestern, heute und morgen bestimmt“ (Nr. 2415). Somit stellen auch die Tiere wie die Pflanzen und Rohstoffe eine Ressource dar, die der Mensch zur Produktion von Nahrungsmitteln, zur Herstellung von Kleidern, bei der Arbeit sowie in der Freizeit und schließlich für die medizinische und wissenschaftliche Forschung nutzen darf (Nr. 2417). Die Rede ist von „vernünftigen Grenzen“ in der Verhältnisbestimmung der Zielsetzung zu den inkaufgenommenen Schäden und Schmerzen für die Tiere sowie davon, dass es „der Würde des Menschen widerspricht, Tiere nutzlos leiden zu lassen und zu töten“ (Nr. 2418). Schließlich solle der Mensch den Tieren „nicht die Liebe zuwenden, die einzig Menschen gebührt“ (ebd.). Insgesamt ergibt sich ein eher ambivalenter Befund, da einerseits die je eigene Güte und Vollkommenheit jedes Geschöpfs anerkannt wird, andererseits aber die schöpfungsgemäße Bestimmung der Tiere zum Wohl der Menschen das Grundmotiv für die Tier-Mensch-Beziehung bildet. Die Tatsache, dass der Umgang des Menschen mit Tieren vielfach von Nutzungsinteressen und Gewalt geprägt ist, wird nicht kritisch reflektiert. Diese Ambivalenz wird durch die mangelnde Präzisierung von wichtigen ethischen Fragen, etwa jene nach den angeführten „vernünftigen Grenzen“, bestärkt (Martin M. Lintner 2020, 28-29).

Eine bedeutsame Entwicklung in der katholischen Lehrtradition finden wir in der Umweltenzyklika Laudato si' (2015) von Papst Franziskus, mit der er sich ausdrücklich nicht nur an die Katholikinnen und Katholiken, auch nicht nur an die Christinnen und Christen, sondern an alle Menschen wendet, denen die Zukunft unserer Erde am Herzen liegt. Er kritisiert die jahrhundertelang vorherrschende anthropozentrische Perspektive, die die Geschöpfe - und damit auch die Tiere - auf ihren instrumentellen Nutzen reduziert oder auf eine technisch-ökonomische Rationalität verengt, als einen „despotischen“ und „fehlgeleiteten“ Anthropozentrismus (Nrn. 68-69, 118-119, 122). Wiederholt spricht er vom „Eigenwert“, den jedes Lebewesen besitzt und den es anzuerkennen gilt (Nrn. 16, 33, 69, 118, 209). Deshalb, so Franziskus, „sagt die Kirche heute nicht einfach, dass die anderen Geschöpfe dem Wohl des Menschen völlig untergeordnet sind, als besäßen sie in sich selbst keinen Wert und wir könnten willkürlich über sie verfügen“ (Nr. 69), und spricht „von einem Vorrang des Seins vor dem 
Nützlichsein"4 (ebd.). Es fällt auf, dass LS von den Geschöpfen und von den Lebewesen im Allgemeinen, nicht jedoch von den Tieren im Speziellen spricht. Es bleibt deshalb offen, was diese Aussagen spezifisch für die Tiere bedeuten und was genau die Anerkennung des Eigenwertes eines jeden Lebewesens für die Mensch-Tier-Beziehung bedeutet. So werden konkrete tierethische Probleme in LS nicht erwähnt, auch nicht jene mit umweltethischer Relevanz wie die industrialisierte Nutztierhaltung. Trotzdem stellt Laudato si' einen wichtigen Impuls dar, an dieser Stelle weiterzudenken und das Desiderat einer christlichen Tierethik zu verwirklichen (Martin M. Lintner 2020).

Der Beitrag der orthodoxen Kirchen besteht weniger in spezifischen Aussagen über die Tiere bzw. zu tierethischen Fragen, sondern darin, das Bewusstsein für die gemeinsame christliche Schöpfungsverantwortung geschärft zu haben, besonders im Rahmen der Europäischen Ökumenischen Versammlungen von Basel (1989), Graz (1997) und Sibiu (2007). .5 Auch die Initiative für eine jährliche Schöpfungszeit im liturgischen Kalender, die in vielen Kirchen im Herbst zwischen dem 1. September und 4. Oktober gefeiert wird, verdankt sich einer Anregung des griechisch-orthodoxen Ökumenischen Patriarchen von Konstantinopel, Bartholomäus I. Tierethik wird in der orthodoxen Theologie jedoch nicht eigens thematisiert, sondern in die Umweltethik integriert, sodass der Verweis auf die Schöpfung als hinreichend angesehen wird, um die Würde der Tiere als Geschöpfe herauszustellen. Ein spezifischer Beitrag der orthodoxen Kirchen zur Umweltethik liegt „in der liturgisch-spirituellen Theologie und Praxis, in der Kosmologie, im Naturverständnis und in der ausgebauten Ethik des Lebens" (Frank Mathwig/Christoph Stückelberger 2007, 160).

4 Der Papst zitiert an dieser Stelle die Deutsche Bischofskonferenz 1980, Zukunft der Schöpfung - Zukunft der Menschheit. Erklärung zu Fragen der Umwelt und der Energieversorgung (Die deutschen Bischöfe 28), Bonn, II, 2.

5 In der Charta Oecumenica, den Leitlinien für die wachsende Zusammenarbeit unter den Kirchen in Europa, die 2001 von der Konferenz Europäischer Kirchen und dem Rat der Europäischen Bischofskonferenzen im Anschluss an die Europäischen Ökumenischen Versammlungen von Basel und Graz verabschiedet worden sind, werden die Tiere nicht eigens genannt, auch nicht im Abschnitt „Die Schöpfung bewahren“, in dem es heißt: „Im Glauben an die Liebe Gottes, des Schöpfers, erkennen wir dankbar das Geschenk der Schöpfung, den Wert und die Schönheit der Natur. Aber wir sehen mit Schrecken, dass die Güter der Erde ohne Rücksicht auf ihren Eigenwert, ohne Beachtung ihrer Begrenztheit und ohne Rücksicht auf das Wohl zukünftiger Generationen ausgebeutet werden.“ 


\section{Interdisziplinäre Annäherungen an eine christliche Tierethik}

In der katholischen und evangelischen theologischen Ethik ist die Tierethik herkömmlich in die Umwelt- oder aber in die Bioethik integriert. Sie etabliert sich aber zusehends als eine eigenständige Disziplin. Mehrere Autorinnen und Autoren haben in den vergangenen Jahren unterschiedliche Ansätze einer christlichen Tierethik erarbeitet (Martin M. Lintner 2018). Dabei zeigt sich, dass die meisten dieser Konzeptionen bei der Frage nach der Bestimmung des moralischen Status der Tiere eine gewisse Nähe zu einem der „klassischen“ umwelt- und bioethischen Lösungsansätze aufweisen. Diese lassen sich in zwei große Gruppen unterscheiden: anthropozentrische und physiozentrische Positionen. Der Anthropozentrismus lässt sich unterteilen in Ansätze, die den Menschen und seine Interessen in den Mittelpunkt stellen und deshalb eine Nutzung der nichtmenschlichen Wirklichkeit inklusive der Tiere für menschliche Interesse für gut heißen. Dieser sogenannte axiologische Anthropozentrismus wird heute weitgehend problematisiert und kritisch in Frage gestellt, da er den Wert von Tieren auf deren Nützlichsein für den Menschen reduziert bzw. den Tieren keinen wie auch immer verstandenen Eigen- bzw. inhärenten Wert zuerkennt, sie also nicht um ihrer selbst willen als schützenswert erachtet. Davon zu unterscheiden sind Formen der aufgeklärten oder gemäßigten Anthropozentrik, die die Verantwortung des Menschen für die Tiere durch seine Vernunftbegabung begründen. Die Nutzung von Tieren wird nicht abgelehnt, aber es werden ihr ethische Grenzen gesetzt. Der Mensch wird in die moralische Pflicht gegenüber Tieren genommen und trägt dafür Verantwortung, in seinem Umgang mit ihnen ihre artspezifischen Bedürfnisse und Fähigkeiten zu berücksichtigen und Prinzipien wie ökologische Nachhaltigkeit und Gerechtigkeit zu befolgen. Die physiozentrischen Positionen umfassen den Pathozentrismus bzw. Sentientismus, den Biozentrismus und den Ökozentrismus. Der Pathozentrismus bzw. Sentientismus stellt die Empfindungsfähigkeit eines Lebewesens als entscheidendes Kriterium für die Bestimmung seines moralischen Status in den Vordergrund, d. h. die Fähigkeit, negative Empfindungen wie Schmerzen und positive wie Freude zu haben bzw. wahrzunehmen. Für den Biozentrismus ist ausschlaggebend, dass etwas lebt, und zwar unabhängig von seiner Empfindungs- oder Vernunftfähigkeit. Albert Schweitzer brachte diese Position in seiner Ethik der "Ehrfurcht vor dem Leben“ pointiert auf den Punkt: „Ich bin Leben, das leben will, inmitten von Leben, das leben will“ (Albert Schweitzer ${ }^{9}$ 2008, 21). Der Ökozentrismus vertritt die Auffassung, dass alles, was auf der Welt existiert, wertvoll und schützenswert ist, entweder als individuelles Seiendes (individueller Ökozentrismus) oder als Teil 
eines Kollektivs (z. B. einer Art) bzw. von komplexen Funktionszusammenhängen (z. B. des Ökosystems). Je nachdem, ob innerhalb dieser Ansätze den verschiedenen Formen von Lebewesen oder unbelebten Dingen je nach Zugehörigkeit zu einer Lebensstufe oder Gruppe ein je unterschiedlicher Wert zuerkannt wird oder nicht, differenziert man zwischen hierarchischen oder egalitären Ansätzen. Während hierarchische Ansätze eine Interessensabwägung aufgrund der Zugehörigkeit zu einer Spezies als ethisch legitim ansehen, wird die Spezieszugehörigkeit in den egalitären Ansätzen nicht als moralisch relevant anerkannt. Allerdings gibt es auch in diesen Positionen Differenzierungen, nach denen z. B. spezifische Eigenschaften auf der sensitiven, emotionalen und kognitiven Ebene in unterschiedlichen Maßen als moralisch relevant gewertet werden.

Es besteht ein breiter Konsens darüber, dass Tiere aus dem Kreis der moralisch zu berücksichtigenden Lebewesen nicht ausgeschlossen werden dürfen. Wie ein roter Faden durchzieht die Diskussionen allerdings nicht nur die Frage nach der Begründung und Reichweite der moralischen Pflichten gegenüber Tieren, sondern auch, mit welcher Terminologie der moralische Status von Tieren angemessen benannt werden kann. Interessanterweise wurde 1986 ins deutsche Tierschutzgesetz ${ }^{6}$ und 1992 in die schweizerische Bundesverfassung7 die religiös konnotierte Begrifflichkeit des Tieres als „Mitgeschöpf“ bzw. der „Würde der Kreatur" aufgenommen, was intensive philosophische, theologische wie auch rechtswissenschaftliche Debatten über die Auslegung, rechtliche Konkretisierung und Justiziabilität dieser Konzepte zur Folge hatte (Philipp Balzer et al. 2001; Carl Friedrich Gethmann 2001; Margot Michel 2012).

Auf drei nach wie vor intensiv diskutierte Fragen soll an dieser Stelle besonders hingewiesen werden. (1) Die erste Frage ist theoretischer Natur: Wie ist die „Würde der Kreatur“8 bzw. die „Tierwürde“ zu verstehen? Viele

6 „Zweck dieses Gesetzes ist es, aus der Verantwortung des Menschen für das Tier als Mitgeschöpf dessen Leben und Wohlbefinden zu schützen. Niemand darf einem Tier ohne vernünftigen Grund Schmerzen, Leiden oder Schäden zufügen“ ( $\mathbb{1}$ ) (Hervorhebung MML).

7 „Der Mensch und seine Umwelt sind vor Missbräuchen der Gentechnologie geschützt. Der Bund erlässt Vorschriften über den Umgang mit Keim- und Erbgut von Tieren, Pflanzen und anderen Organismen. Er trägt dabei der Würde der Kreatur sowie der Sicherheit von Mensch, Tier und Umwelt Rechnung und schützt die genetische Vielfalt der Tier- und Pflanzenarten“ (Art. 120) (Hervorhebung MML).

8 Nicht eingegangen werden kann an dieser Stelle auf die spannende Frage, welche Konsequenzen diese Terminologie auf den Umgang mit den Pflanzen hat, die als Lebewesen ebenso Kreaturen bzw. Mitgeschöpfe sind (Sabine Odparlik et al. [Hg.] 2008; Sabine Odparlik 2013). 
Publikationen setzen sich mit der Problematik auseinander, welches Verhältnis zwischen der Würde von Menschen und jener von Tieren besteht (Alberto Bondolfi et al. [Hg.] 1997; Hans J. Münk 1997; Heike Baranzke 2002; Sabine Odparlik/Peter Kunzmann [Hg.] 2007 u. a. m.). Kann oder soll "Würde der Kreatur" unterschiedslos auf menschliche wie nichtmenschliche Tiere bezogen werden, was letztlich zur Anerkennung von Tierindividuen als Personen führt? Dies fordern Tierrechtspositionen, die konsequenterweise vertreten, dass Tiere (wenn auch in abgestufter Form) Ansprüche auf grundlegende Rechte, Freiheiten und Schutz haben (Sue Donaldson/Will Kymlicka 2013). Die Verwendung von zumal empfindungsfähigen Tieren für menschliche Zwecke wäre dann abzulehnen (Gary L. Francione/Anna Charlton 2015). Oder ist der Begriff im analogen Sinn zu verwenden, sodass die spezifische Menschenwürde von der kreatürlichen Würde der Tiere zu differenzieren ist (Heike Baranzke 2002)? Darauf bauen unterschiedliche Tierschutzpositionen auf. Auch wenn sie sich gegen den Vorwurf des Speziesismus zu verteidigen haben (Eberhard Schockenhoff 2009, 149-152), lassen diese Positionen eine Abwägung menschlicher und tierlicher Interessen zu, jedoch unter der Bedingung, dass Tiere nicht ausschließlich auf ihren Nutzwert reduziert, sondern ihre artspezifischen Grundbedürfnisse respektiert und erfüllt werden (Martin M. Lintner 2017 u. a. m.). (2) Auf eine zweite Frage sei hier nur hingewiesen - gleichsam in Klammern, da sie im vorliegenden Band nicht eigens thematisiert wird, obwohl sie in diversen Beiträgen unverkennbar eine untergründige Rolle spielt. Sie betrifft die linguistische Herausforderung, wie von Menschen und Tieren gesprochen werden soll, um einerseits die Nähe und Ähnlichkeit zwischen beiden Seiten, andererseits auch Unterschiede und Grenzen zu benennen. Ist die Rede von „menschlichen und nichtmenschlichen Tieren (oder Lebewesen)“ angemessen? Oder jene von „menschlichen und anderen Tieren (oder Lebewesen)“, um die Tiere nicht negativ durch ihr Nicht-Menschsein zu definieren? Und wie kann sprachlich sichtbar gemacht werden, dass zwischen einigen tierlichen und der menschlichen Spezies eine viel größere Nähe besteht als zwischen den meisten tierlichen? Zu problematisieren sind ebenso Ausdrucksformen, die bestimmte Tiere linguistisch auf ihre Funktion reduzieren - beispielsweise Nutz-, Arbeits- oder Versuchstiere, Zucht-, Mast- oder Schlachtvieh und die die damit verbundene Nutzung und menschliche Macht- und Gewaltausübung über sie bereits sprachlich legitimieren (Reinhard Heuberger 2017 und 2021). Manche Autorinnen und Autoren - auch des vorliegenden Bandes - verwenden bewusst das Adjektiv "tierlich“ anstelle von „tierisch“, welches zumindest in manchen Sprechkontexten einen abwertenden Unterton hat. (3) Die dritte Frage hingegen ist mehr praktischer 
Natur: Haben tierschutzrechtliche Bestimmungen sowie die Anerkennung der Tiere als Mitgeschöpfe bzw. das Konzept der "Würde der Kreatur“ einen wirkungsvolleren rechtlichen Schutz der Tiere und ihrer Interessen zur Folge? Die Realität ist ernüchternd. Besonders im Kontext der agrarischen Nutztierhaltung unterbleibt eine ernsthafte Bekämpfung von systematischen strafbaren Verletzungen des Tierschutzrechts immer noch weitgehend, während vorwiegend lediglich individuelles Fehlverhalten in Einzelfällen strafrechtlich geahndet wird (Jens Bülte 2018; Annabelle Thilo 2020, 172-294). Verschiedene Initiativen machen auf diese Missstände aufmerksam und verlangen sowohl rechtliche als auch systemische Veränderungen, so z. B. die Initiative gegen Massentierhaltung in der Schweiz (2019), das Tierschutzvolksbegehren in Österreich (2021) oder der Untersuchungsausschuss zu Tiertransporten des EU-Parlamentes (2020-2021).

Kehren wir zurück zu den tierethischen Positionen. Eine ausschließliche Zuordnung der verschiedenen theologischen tierethischen Entwürfe $\mathrm{zu}$ je einem der weiter oben aufgezeigten anthropo- bis ökozentrischen Modelle, die sich zwischen den Polen von Tierrechts- auf der einen und Tierschutzpositionen auf der anderen Seite bewegen, ist wenig zielführend. Jeder dieser stichwortartig vorgestellten Lösungsansätze vertritt nämlich berechtigte Anliegen und entspricht auch je unterschiedlichen moralischen Intuitionen, weist zugleich aber auch Grenzen auf bzw. beinhaltet kritische Aspekte. Es geht also nicht um eine strikte Abgrenzung dieser Ansätze voneinander, sondern - auch im Sinne einer legitimen Pluralität und Bandbreite von Perspektiven - um eine kritische wechselseitige Ergänzung.

Zudem knüpfen die unterschiedlichen theologisch-ethischen Ansätze an verschiedene moralphilosophische tierethische Konzeptionen an. Vor allem seit den 1970er-Jahren, im Besonderen in Folge der Publikation von Peter Singers Animal Liberation (1975) wurde eine Vielzahl von alternativen Konzepten entfaltet: utilitaristische Ansätze, Theorien moralischer Rechte und Würde, vertragstheoretische Konzeptionen, Mitleidsethik und Ethik der Fürsorge, tugendethische und schließlich multikriterielle Ansätze (Ursula Wolf [Hg.] 2016, 11; Friederike Schmitz 22017, 1-13). Die Spannweite reicht von Ansätzen, die motiviert von moralischer Empörung über den oft ausbeuterischen und gewaltsamen Umgang mit Tieren, zumal in der Nutztierhaltung und in der biomedizinischen und militärischen Forschung, stark emotiv-appellativ geprägt sind, bis hin zum Ringen um rational begründete Theorien als belastbare Basis für die ethische Beurteilung sowie Abwägung von menschlichen und tierlichen Interessen in konkreten Anwendungsbereichen. Die legitime Bandbreite unterschiedlicher Ansätze findet sich - wenig verwunderlich - auch in der theologischen 
Ethik wieder. Wie im Kontext der philosophischen Ethik wird Tierethik zudem zusehends nicht nur als eine Bereichsethik der angewandten Ethik verstanden, da die Frage der Tier-Mensch-Beziehung neben den Grundfragen der Ethik auch ganz grundsätzlich das Selbstverständnis des Menschen sowie die unterschiedlichsten Bereiche von Ökologie, Ökonomie, Medizin, Forschung usw. bis hin zu Fragen der Nachhaltigkeit und sozialen Gerechtigkeit betrifft. Tiere spielen in all diesen Bereichen eine Rolle (Herwig Grimm/Markus Wild 2016, 16).

Nach diesem überblicksmäßigen und keineswegs erschöpfenden Aufriss von unterschiedlichsten Themen und Diskursen, mit denen sich theologisch-ethische Tierethikansätze auseinanderzusetzen haben, sollen abschließend kurz die Gliederung und die Inhalte der einzelnen Beiträge der vorliegenden Publikation vorgestellt werden.

\section{Inhalt und Gliederung des vorliegenden Bandes}

Der erste Band der Reihe Interdisziplinäre Tierethik (ITE) / Interdisciplinary Animal Ethics (IAE) beleuchtet unterschiedliche Facetten, die eine christliche Tierethik zu bedenken hat. Wie der Untertitel zum Ausdruck bringt, geht es um interdisziplinäre Annäherungen, nicht um den Entwurf einer einheitlichen Theorie bzw. einer homogenen tierethischen Position im Singular. Naturwissenschaftliche, philosophische, biblische und theologische Ressourcen und Aspekte sollen benannt werden. Zu Wort kommen deshalb Vertreterinnen und Vertreter nicht nur von verschiedenen wissenschaftlichen Disziplinen, sondern auch - wie aus der Lektüre der diversen Beiträge deutlich wird - von unterschiedlichen tierethischen Positionen, wobei der Fokus der einzelnen Artikel nicht darauf liegt, die je eigene Position darzustellen und stark zu machen.

Das erste Kapitel dient einer Standortbestimmung aus historischer wie gegenwärtiger Perspektive. Heike BARANZKE liefert eine ideengeschichtliche Verortung unterschiedlicher tierethischer Ansätze, die noch die heutigen Tierethikdebatten grundieren. Sie bietet einen historischen Überblick über die Reflexion auf die Mensch-Tier-Beziehung von der Antike bis in die Neuzeit und zeigt auf, wie ein überwiegend antagonistisch gegeneinander ausgespieltes Gleichheits- und Differenzdenken in abendländischen Gerechtigkeitskonzepten die klassischen tierethischen Positionen prägen. Dabei arbeitet sie am Beispiel von Porphyrios aus Tyros und Immanuel Kant heraus, wie inhomogene Mensch-Tier-Gemeinschaften moralphilosophisch integrativ gedacht werden können, indem begründungstheoretische von anwendungsethischen Argumentationen unterschieden 
werden. Susana MONSÓ, Andreas AIGNER und Herwig GRIMM führen anhand einer kritischen Auseinandersetzung mit dem Argument des moralischen Individualismus, wonach individuelle Fähigkeiten und Eigenschaften, nicht jedoch die Zugehörigkeit zu einer Gruppe oder Spezies ausschlaggebend dafür sind, wie ein Individuum behandelt werden soll, in die gegenwärtigen tierethischen Debatten ein. Dabei analysieren sie die in der Tradition Wittgensteins stehende Kritik von Alice Crary und Cora Diamond an „orthodoxen“ tierethischen Ansätzen, für die z. B. die Empfindungsfähigkeit oder die Fähigkeit, Interessen haben zu können, von zentraler Bedeutung sind (Peter Singer, Tom Regan u. a.). Trotz der Kritik am traditionellen moralischen Individualismus argumentieren die Autorin und die Autoren dafür, das Individuum in der Ethik nicht gänzlich außer Acht zu lassen, und plädieren für eine Ethik, welche spezifische Eigenschaften (menschliche wie tierliche) thematisieren kann und dabei der Reichhaltigkeit und Komplexität Beachtung schenkt, die das moralische Leben charakterisieren.

Im zweiten Kapitel wird auf dem Hintergrund ethologischer Erkenntnisse der naturwissenschaftliche und tierphilosophische Paradigmenwechsel im Blick auf die Tiere aufgezeigt. Kurt KOTRSCHAL zeigt stammesgeschichtliche Gemeinsamkeiten zwischen Menschen und Tieren auf und stellt neuere evolutionsbiologische Erkenntnisse über emotionale, soziale und kommunikative Fähigkeiten von Tieren vor. Zugleich räumt der Autor ein, dass es menschliche Alleinstellungsmerkmale gibt, z. B. die menschliche Fähigkeit zur Symbolsprache und zu abstraktem Denken, auch wenn er diese aus Sicht eines Evolutionsbiologen nicht als ,anthropologische Differenz" gewertet wissen will. Damit wirft er exemplarisch die weiterführende Frage auf, wie naturwissenschaftliche Erkenntnisse geisteswissenschaftlich zu deuten sind. Markus WILD hingegen beschäftigt sich mit der moralphilosophischen Bedeutung von Kognition und Bewusstsein bei Tieren. Am Beispiel von Hühnern, Hähern und Fischen zeigt er auf, dass Tiere über intentionale Zustände und Empfindungen verfügen. Nach der repräsentationalistischen Theorie des Bewusstseins lassen diese auf Intentionalität und Bewusstsein schließen.

Das dritte Kapitel ist biblischen und theologischen Weichenstellungen gewidmet, die aufgrund von neuen exegetischen Einsichten sowie von theologischen Reflexionen über die Tiere vorgenommen werden müssen. Ute NEUMANN-GORSOLKE beleuchtet ethische Aspekte der MenschTier-Beziehung im Alten Testament, welche durch die existentielle Bezogenheit des Menschen auf die Tiere und von Wertschätzung gegenüber den Tieren geprägt war. Für eine egalitaristische Mensch-Tier-Beziehung und eine entsprechende Ethik, so die Autorin, würden die alttestamentli- 
chen Texte jedoch keine Grundlage liefern. Julia ENXING fragt nach dem Platz der Tiere in der gegenwärtigen Schöpfungstheologie und -spiritualität. Zunächst konstatiert sie, dass Tieren für die Artikulation einer theologischen Anthropologie und Schöpfungstheologie nicht nur in der Tradition, sondern auch in den gegenwärtigen einschlägigen Publikationen keine Bedeutung zugemessen wird. Sie plädiert für eine ganzheitlich gedachte Schöpfungstheologie - im Sinne einer planetarischen Solidarität - sowie für eine Anthropologie, die den Menschen in seiner Abhängigkeit von der Ko-Schöpfung begreift. Peter KUNZMANN behandelt die neuralgische Frage nach dem Verständnis von „Tierwürde“. Er zieht die Linien von einer religiös-metaphysischen Wertschätzung der Tiere als „Geschöpfe“ bis zu ihrem Rang in der Enzyklika Laudato si, argumentiert jedoch, dass Konzeptionen der Würde - unter Berücksichtigung der Analogie des Begriffs - geeigneter sind als die Begrifflichkeit der Kreatur, um das ,je Eigene" der Tiere zu benennen, das sie einerseits schützenswerter macht als andere Naturdinge, andererseits eine Differenzierung zur Würde des Menschen zulässt. Jakob OHM untersucht in seinem Beitrag, inwieweit aus Sicht der theologischen Ethik eine Vermittlung der eminent theologischen Begriffe Sakralität und Person einen unterbewussten Vorschub für eine anthropozentrische Hybris des Menschen in ethischen Fragestellungen leistet oder aber zu einem tieferen Verständnis der Stellung des Menschen im Zusammenleben mit anderen Geschöpfen beitragen kann. Julia BLANC fragt, ausgehend von den vielfältigen Formen von Leid, das Tieren zugefügt wird, ob sich im sozialethischen wie theologischen Prinzip der Option für die Armen ein Anschlusspunkt nicht nur für eine christliche Tierethik, sondern letztlich auch für eine neue Tiertheologie findet. Martin M. LINTNER untersucht die Bedeutung der Tiere in der Liturgie. Diese stellt in der Art und Weise, wie sie das Leben integriert, im Licht der Heiligen Schrift reflektiert und zur Sprache bringt, einen „ethischen Lernort“ dar. Ebenso arbeitet er die liturgisch relevante Bedeutung der Tiere aus schöpfungstheologischer wie heilsgeschichtlicher Perspektive heraus und plädiert für thematische Gottesdienste und Votivmessen. Schließlich geht er auf die Frage ein, wie sinnvoll Gottesdienste mit Tieren sind.

Im vierten Kapitel werden tierethische Herausforderungen im Kontext systemischer Zusammenhänge reflektiert. Markus VOGT untersucht die agrarökonomischen und -politischen Aspekte der Tierethik im Spannungsfeld von Tierwohl, Landwirtschaft und Agrarpolitik. Er diagnostiziert einen erheblichen Mangel an sozialethischer Auseinandersetzung mit den Systembedingungen der Nutztierhaltung in der modernen Landwirtschaft sowie des damit verknüpften Verlustes von Biodiversität. Anita IDEL zeigt die auch aus tierethischer Sicht relevanten agrarökologischen Potenziale 
nachhaltiger Beweidung für Bodenfruchtbarkeit und Klima auf. Da Landwirtschaft nur eine Zukunft hat, wenn sie in fruchtbaren Landschaften gedacht und behandelt wird, fordert die Autorin die Überwindung eines „kranken Naturbegriffs" der einseitigen Konkurrenz hin zur biologischen und sozialen Kooperation. Matthias GAULY geht auf die gegenwärtigen politischen Debatten und kritischen Anfragen an die Landwirtschaft ein, in denen Fragen zu Tierwohl und Tiergesundheit eine zunehmend wichtige Rolle spielen. Er stellt Kriterien vor, wie man Letztere bewerten kann, und untersucht, unter welchen Bedingungen positive Veränderungen in der Nutztierhaltung möglich sind. Michael ROSENBERGER gibt aus einer vertragstheoretischen Perspektive eine Antwort auf die tierethische „Gretchenfrage“, ob wir Tiere töten und essen dürfen. Er argumentiert, dass Systeme der Tiernutzung einschließlich der Tiertötung dann, wenn sie gewissen Gerechtigkeitsstandards entsprechen, „ähnlich gerecht“ sind wie die freie Wildbahn und die Ressourcen der Erde „halbwegs fair“ zwischen menschlichen und nichtmenschlichen Tieren verteilen. Kurt REMELE hingegen problematisiert von einem sentientistischen Zugang her nicht nur das Essen von Fleisch und den Konsum anderer tierlicher Produkte, sondern auch die Herstellung von Kleidung und anderen Artefakten aus tierlichen Materialien. In Anlehnung an den Roman „Das Leben der Tiere" von J. M. Coetzee bewertet er den Unterschied zwischen Essen von Fleisch und Tragen von Leder als lediglich „unterschiedliche Grade von Obszönität“. Auch Martina BESLER geht davon aus, dass die gegenwärtige Situation des Konsums tierlicher Produkte vielfach ethisch nicht zu vertreten ist, und sieht in der Veränderung der Lebensgewohnheiten im Blick auf den Umgang mit tierlichen Produkten eine dringliche moralpädagogische Herausforderung für die persönliche Gewissensbildung. Sie wählt hierfür anhand der Gewissenslehre des spanischen Jesuiten Juan Azor (1535-1603) einen tugendethischen Zugang. Matthias EGGEL erläutert die Richtlinie des Europäischen Parlaments und des Rates der Europäischen Union zum Schutz der für wissenschaftliche Zwecke verwendeten Tiere. Er zeigt auf, dass die Prüfung auf wissenschaftliche Erforderlichkeit, Eignung und Verhältnismäßigkeit drei Eckpfeiler des Genehmigungsverfahrens darstellen, welche laut Gesetz erfüllt sein müssen, damit ein Tierversuch ethisch vertretbar ist, und benennt ihre Probleme sowie die Beschränkung ihrer „ethischen Reichweite“.

Das fünfte Kapitel beinhaltet schließlich zwei Beiträge zu zwei exemplarischen tierethischen Problemfeldern. Katharina EBNER untersucht, über die herkömmlichen, vorwiegend medizinethischen Diskussionen hinausgehend, die mit der Xenotransplantation dringlich werdenden tierethischen Anfragen an das Mensch-Tier-Verhältnis. Sie stellt ausgewählte alter- 
native Ordnungskategorien vor und nimmt dabei wahr, dass das MenschTier-Verhältnis gegenwärtig Gegenstand von Neuaushandlungen ist. Diese ergeben sich aus dem zunehmenden Wissen über Tiere, aber auch aus der Veränderung von Sinnhorizonten, weil die Menschen die Gestaltungskraft und ihr Einwirken auf die gesamte Umwelt mehr und mehr ausweiten. Religiös begründete Ermächtigungsmodelle hierfür werden in einer säkularen Gesellschaft jedoch zusehends in Frage gestellt. Christina POTSCHKA betritt Neuland und untersucht aus tierethischer Perspektive den Einsatz von Roboter-Tieren mit künstlicher Intelligenz im therapeutischen und pflegerischen Bereich. Sie analysiert, dass es vergleichbar mit dem Anthropomorphismus auch einen „Animorphismus“ gibt, d. h. die Übertragung tierlicher Eigenschaften auf nichttierliche Roboter, und fragt anhand von tierethischen Ansätzen, ob bzw. inwiefern Roboter-Tieren in irgendeiner Form ein moralischer Wert zuerkannt werden soll.

\section{Literatur}

Balzer, Philipp/Rippe, Klaus Peter/Schaber, Peter 2001, Menschenwürde vs. Würde der Kreatur. Begriffsbestimmung, Gentechnik, Ethikkommissionen (Alber-Reihe Philosophie), Freiburg i. Br./München.

Baranzke, Heike 2002, Würde der Kreatur? Die Idee der Würde im Horizont einer integrativen Bioethik (Epistemata. Würzburger Wissenschaftliche Schriften/ Reihe Philosophie 328), Würzburg.

Bondolfi, Alberto/Pezzoli-Olgiati, Daria/Lesch, Walter (Hg.) 1997, „Würde der Kreatur". Essays zu einem kontroversen Thema (Theophil 5), Zürich.

Bülte, Jens 2018, Zur faktischen Straflosigkeit institutionalisierter Agrarkriminalität, in: Goltdammer's Archiv für Strafrecht 165 (1), 35-56.

Bundesverfassung der Schweizerischen Eidgenossenschaft, in: https://www.admin.c h/opc/de/classified-compilation/19995395/index.html (abgerufen am 31.10.2020).

Deutsche Bischofskonferenz 1993, Die Verantwortung des Menschen für das Tier. Positionen - Überlegungen - Anregungen (Arbeitshilfen 113), Bonn.

Deutsches Tierschutzgesetz, in: https://www.gesetze-im-internet.de/tierschg/BJNR0 12770972.html (abgerufen am 05.07.2020).

Dietrich, Hans-Eberhard 2019, Gegen den Strom, in: Seidel, Ulrich/Dietrich, HansEberhard 2019, An der Seite der Tiere. Christsein und ein neuer Umgang mit der Kreatur (Christsein aktuell 5), Münster, 95-109.

Donaldson, Sue/Kymlicka, Will 2013, Zoopolis. Eine politische Theorie der Tierrechte, Berlin. 
Enxing, Julia 2021, Schöpfungstheologie im Anthropozän. Gedanken zu einer planetarischen Solidarität und ihrer (theo)politischen Relevanz [Beitrag im vorliegenden Band].

Evangelische Kirche in Deutschland 1991, Zur Verantwortung des Menschen für das Tier als Mitgeschöpf (EKD-Texte 41), Hannover.

Evangelische Kirche in Deutschland 2019, Nutztier und Mitgeschöpf! Tierwohl, Ernährungsethik und Nachhaltigkeit aus evangelischer Sicht (EKD-Texte 133), Hannover.

Francione, Gary L./Charlton, Anna 2015, Animal Rights. The abolitionist Approach, Logan.

Franziskus 2015, Laudato si'. Enzyklika über die Sorge für das gemeinsame Haus (24.05.2015).

Gethmann, Carl Friedrich 2001, Tierschutz als Staatsziel - Ethische Probleme, in: Thiele, Felix (Hg.) 2001, Tierschutz als Staatsziel? Naturwissenschaftliche, rechtliche und ethische Aspekte (Europäische Akademie/Graue Reihe 25), 50-76.

Grimm, Herwig/Wild, Markus 2016, Tierethik zur Einführung, Hamburg.

Hagencord, Rainer ${ }^{4}$ 2009, Diesseits von Eden. Verhaltensbiologische und theologische Argumente für eine neue Sicht der Tiere, Regensburg.

Heuberger, Reinhard 2017, Sprachgebrauch: Das Mensch-Tier-Verhältnis aus linguistischer Sicht, in: Kompatscher, Gabriela/Spannring, Reingard/Schachinger, Karin 2017, Human-Animal-Studies (utb 4759), Münster/New York, 48-53.

Heuberger, Reinhard 2021, Die Rolle der Sprache im Mensch-Tier Verhältnis unter besonderer Berücksichtigung des Bildungsbereichs. Eine linguistische Perspektive, in: Horstmann, Simone (Hg.) 2021, Interspezies Lernen. Grundlinien interdisziplinärer Tierschutz- und Tierrechtsbildung (Human-Animal Studies), Bielefeld [in Druck].

Horstmann, Simone 2020, Was fehlt, wenn uns die Tiere fehlen? Eine theologische Spurensuche, Regensburg.

Horstmann, Simone (Hg.) 2021, Religiöse Gewalt an Tieren. Interdisziplinäre Diagnosen zum Verhältnis von Religion, Speziesismus und Gewalt (Human-Animal Studies), Bielefeld [in Druck].

Horstmann, Simone/Ruster, Thomas/Taxacher, Gregor 2018, Alles, was atmet. Eine Theologie der Tiere, Regensburg.

Jung, Martin H. (Hg.) 2002, Christian Adam Dann/Albert Knapp: Wider die Tierquälerei. Frühe Aufrufe zum Tierschutz aus dem württembergischen Pietismus (Kleine Texte des Pietismus 7), Leipzig.

Katechismus der Katholischen Kirche. Neuübersetzung aufgrund der Editio Typica Latina 2003, Vatikan/München.

Konferenz Europäischer Kirchen/Rat der Europäischen Bischofskonferenzen 2001, Charta Oecumenica. Leitlinien für die wachsende Zusammenarbeit unter den Kirchen in Europa, in: http://www.oekumene.at/dl/uuopJKJKmnLJqx4KJK/Cha rta_Oecumenica.pdf (abgerufen am 05.07.2020). 
Lintner, Martin M. 2017, Der Mensch und das liebe Vieh. Ethische Fragen im Umgang mit Tieren. Mit Beiträgen von Christoph J. Amor und Markus Moling, Innsbruck.

Lintner, Martin M. 2018, Einfach zum Fressen gern. Tiere in der theologischen Ethik, in: Herder Korrespondenz 72, 28-31.

Lintner, Martin M. 2020, Respect for the Proper Value of Each Creature. An Animal-Ethical Rethinking of the Encyclical Laudato si', in: Louvain Studies 43 (1), 26-48.

Mathwig, Frank/Stückelberger, Christoph 2007, Grundwerte. Eine theologischethische Orientierung, Zürich.

Michel, Margot 2012, Die Würde der Kreatur und die Würde des Tieres im schweizerischen Recht. Eine Standortbestimmung anlässlich der bundesgerichtlichen Rechtsprechung, in: Natur und Recht 34 (2), 102-109; DOI: 10.1007/ s10357-012-2214-3.

Münk, Hans J. 1997, „Die Würde des Menschen ist unantastbar“ (Art. 1,1 GG). Und was ist mit der Würde der Natur? Theologisch-ethische Überlegungen zur Grundkonzeption einer ökologischen Ethik, in: Verein der Freunde und Förderer des Zentrums für Umwelt und Kultur Benediktbeuern (Hg.) 1997, Handeln für die Zukunft der Schöpfung, München, 29-40.

Odparlik, Sabine 2013, Die Würde der Pflanze. Ein sinnvolles ethisches Prinzip im Kontext der Grünen Gentechnik? (Angewandte Ethik 12), Freiburg i. Br.

Odparlik, Sabine/Kunzmann, Peter (Hg.) 2007, Eine Würde für alle Lebewesen? (ta ethica 4), München.

Odparlik, Sabine/Kunzmann, Peter/Knoepffler, Nikolaus (Hg.) 2008, Wie die Würde gedeiht. Pflanzen in der Bioethik (ta ethica 6), München.

Remele, Kurt 2016, Die Würde des Tieres ist unantastbar. Eine neue christliche Tierethik, Kevelaer.

Schleißing, Stefan/Grimm, Herwig 2012, Tierethik als Thema der Theologie und des kirchlichen Handelns, in: Evangelische Kirche in Deutschland u. a. (Hg.) 2012, Dokumente zum kirchlichen Zeitgeschehen (Kirchliches Jahrbuch für die Evangelische Kirche in Deutschland 2010), Gütersloh, 45-86.

Schmitz, Friederike 22017, Tierethik, Münster.

Schockenhoff, Eberhard 2009, Ethik des Lebens. Grundlagen und neue Herausforderungen, Freiburg i. Br. etc.

Schweitzer, Albert ${ }^{9} 2008$, Die Ehrfurcht vor dem Leben. Grundtexte aus fünf Jahrzehnten, hg. von Hans Walter Bähr, München.

Seidel, Ulrich 2017, Alles, was lebt, ist dein Nächster (Gandhi), in: Hagencord, Rainer/Kretzschmar, Helga (Hg.) 2017, Das Tier in Religion, Kultur und Ethik. Neue Wahrnehmung des Tieres in Theologie und Spiritualität (Jahrbuch Theologische Zoologie 2), Münster, 55-72.

Seidel, Ulrich/Dietrich, Hans-Eberhard 2019, An der Seite der Tiere. Christsein und ein neuer Umgang mit der Kreatur (Christsein aktuell 5), Münster. 
Thilo, Annabelle 2020, Die Garantenstellung des Amtstierarztes. Unter besonderer Berücksichtigung der rechtsphilosophischen und empirischen Implikationen von $\$ 17$ Tierschutzgesetz (Studien zum Strafrecht 106), Baden-Baden.

Wolf, Ursula (Hg.) 2016, Texte zur Tierethik (Reclams Universal-Bibliothek 18535), Stuttgart. 